\title{
EFFICACY OF INTRA-ARTERIAL LIDOCAINE FOR PAIN CONTROL RESULTING FROM TRANSARTERIAL CHEMOEMBOLIZATION OF HEPATOCELLULAR CARCINOMA
}

\author{
Ghulam Abbas, Atif Sheraz, Raza Rahim \\ Armed Forces Institute of Radiology and Imaging / National University of Medical Sciences (NUMS) Rawalpindi Pakistan
}

\begin{abstract}
Objective: To assess the efficacy of intra-arterial lidocaine in peri \& post-procedural pain control and the dose of narcotic analgesic required in hepatocellular carcinoma patients undergoing transarterial chemoembolization.

Study Design: Comparative prospective study.

Place and Duration of Study: Armed Forces Institute of Radiology and Imaging Rawalpindi, from Jan to Jun 2019.

Methodology: A total of 60 patients included in this study where 42 males and 18 were females, age range $45-85$ years who underwent transarterial chemoembolization for hepatocellular carcinoma, were included in the study. patients were equally divided into two groups, group a (30 patients) who underwent transarterial chemoembolization, received 60 mg of intraarterial lidocaine each and group $b$ (30 patients) who underwent transarterial chemoembolization, intra-arterial lidocaine was substituted with normal saline.degree of post-procedural pain was assessed using a subjective method (visual analogue scales score) and an objective method (amount of post-procedural analgesics).

Results: Average peri-procedure visual analogue scale score was 5.06 in group A patient versus 7.2 for those in group B patients $(p=0.037)$. Post-procedure visual analogue scale score in the group A was $2.7 \pm 0.520$ and that for group B was 4.2 \pm 0.761 ( $p=0.025)$. Mean of total dose of nalbuphine in group A was $4.96 \pm 0.764 \mathrm{mg}$ versus $8.3 \pm 1.34 \mathrm{mg}$ for patients in group B $(p=0.036)$. Average length of post procedure hospital stay was $0.9 \pm 0.203$ and $1.41 \pm 0.373$ days for group A and group B respectively $(p=0.002)$.

Conclusion: Intra-arterial administration of lidocaine before infusing the embolization particles for transarterial chemoembolization in patients with hepatocellular carcinoma is safe and effective in doses as low as 50 mg for reducing peri \& postprocedural pain and reducing dosage of narcotic analgesics.
\end{abstract}

Keywords: Hepatocellular carcinoma (HCC), Intra-arterial, lidocaine, Pain control and pes (post embolization syndrome), Trans arterial chemoembolization (TACE) \& visual analogue score (VAS).

This is an Open Access article distributed under the terms of the Creative Commons Attribution License (https://creativecommons.org/licenses/by-nc/4.0/), which permits unrestricted use, distribution, and reproduction in any medium, provided the original work is properly cited.

\section{INTRODUCTION}

Transarterial chemoembolization (TACE) is a well-known technique for the management of unresectable hepatocellular carcinoma. TACE may be used as a neoadjuvant and bridging to resection or orthotopic liver transplantation ${ }^{1}$. In most institutions TACE is considered as an option when the patient is not a surgical candidate for the treatment of hepatocellular carcinoma $(\mathrm{HCC})^{2-7}$. It is indicated as a palliative treatment and considered as the first line therapy for intermediate stage hcc according to the recommendation of american association for study of liver diseases based on randomized controlled trials ${ }^{8}$. TACE is simply administration of cytotoxic drugs with or without lipiodol, by means of a catheter positioned in the tumor supplying hepatic artery followed by the administration of embolizing agents such as spherical gelatin or polyvinyl alcohol particles ${ }^{9}$. In most patients TACE commonly causes procedure-related abdominal pain

Correspondence: Dr Ghulam Abbas, Classified Radiologist, Armed Forces Institute of Radiology \& Imaging, Rawalpindi Pakistan

Received: 25 Jul 2020; revised received: 01 Sep 2020; accepted: 10 Sep 2020 either during or after the procedure, sometimes even in patients who did not experience pain during the procedure. The pain is severe enough to necessitate narcotic analgesia. Post embolization syndrome (PES) is a common complication after embolic procedures and it is a frequent cause of extended inpatient hospital admissions. PES is a self-limited constellation of symptoms consisting of fever, unremitting nausea, general malaise, loss of appetite, and variable abdominal pain following the procedure. Although a definite cause is unknown, this syndrome is thought to be a result of therapeutic cytotoxicity, tumor ischemia and resulting intrahepatic and extrahepatic inflammation ${ }^{10}$.

\section{METHODOLOGY}

This comparative prospective study was conducted at armed forces institute of radiology and imaging (AFIRI) Rawalpindi. It was approved by institutional ethics committee-IERB approval certificate number is 00012. prior to the procedure informed consent was obtained from all of the patients included in study.

The sample size calculated was 60 patients with hepatocellular carcinoma using the who sample size 
calculator, keeping the confidence level 1.96 (95\%), margin of error 0.49 , baseline level of indicators 0.5 , design effect 1.5, expected response rate 0.8 and number of age/sex estimates ${ }^{8}$. Simple random sampling technique was used in this study.

Inclusion criteria for TACE were corrected coagulopathy, single or multifocal HCC, tumor volume $<50 \%$ of liver, patients with child A or B, no main portal vein tumor thrombus and no extrahepatic disease.

Inclusion criteria for administration of lidocaine were same as that of TACE.

Exclusion criteria of TACE were uncorrected coagulopathy, infiltrative type of HCC, tumor volume $>50 \%$ of the liver, patients with child-C according to child pugh classification, extra hepatic metastasis of HCC that was confirmed by CT chest, abdomen and pelvis and thrombosis of main portal vein.

Exclusion criteria for administration of intraarteria lidocaine were patients on pre-existing regular analgesics, patients who required conscious sedation and any contra-indication to lidocaine like heart block.

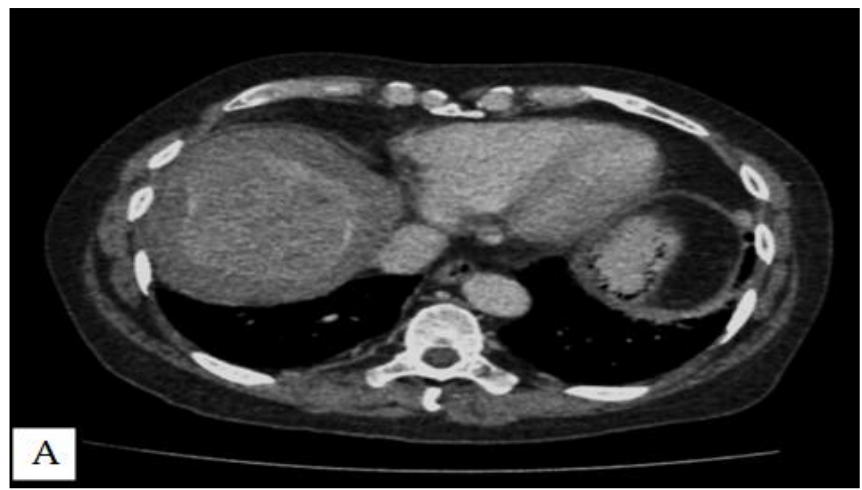

All patients were subjected to standard evaluation for TACE in HCC. The diagnosis was made either by typical imaging criteria of hcc with triphasic dynamic contrast studies by CT (fig-1) or MRI in addition to serum level of alfa fetoprotein or in equivocal cases by of histopathology. Pre-procedural standard investigations for TACE to assess hepatic and renal function and coagulation status were done.

Continuous monitoring of blood pressure, pulse and ECG was done during the entire procedure. TACE was performed after visceral angiography to evaluate arterial supply of the HCC and evaluate patency of portal vein. HCC arterial supply was accessed by selective catheterization using standard $5 \mathrm{fr}$ catheter or $2.8 \mathrm{fr}$ coaxial technique using progreat microcatheter (terumo). once the catheter was in suitable position, a slow injection of doxorubicin $50 \mathrm{mg}$ mixed with $10 \mathrm{ml}$ of lipiodol (ultra-fluids guerbet france) thus forming total volume of chemotherapeutic emulsion was about 15 $\mathrm{ml}$ was administered (fig-2). In some cases, deb-TACE was performed in which $50 \mathrm{mg}$ doxorubicin was mixed with $25 \mathrm{mg}$ hepasphere microsphere 30-60 mic-rons

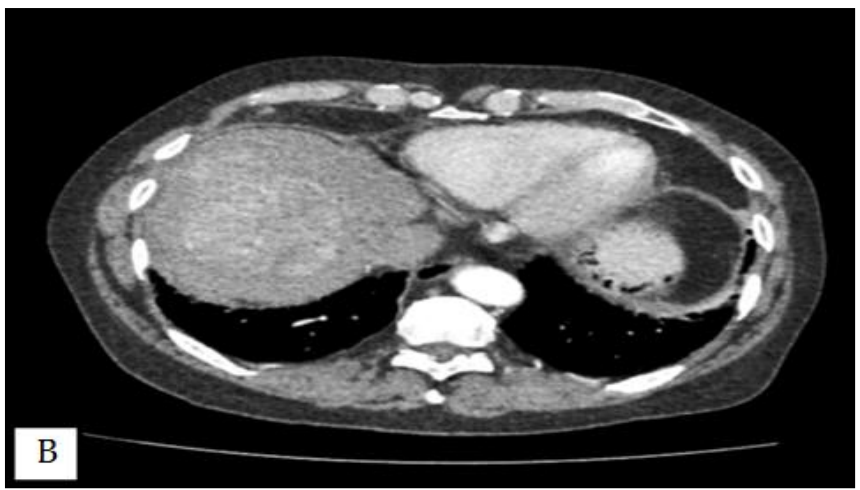

Figure-1: a) Contrast enhaned triphasic CT liver showed segment VIII lesion showing arterial phase enhamcement, b) and washout on portal venous pahse: Consistan with hepatocellular carcinoma.
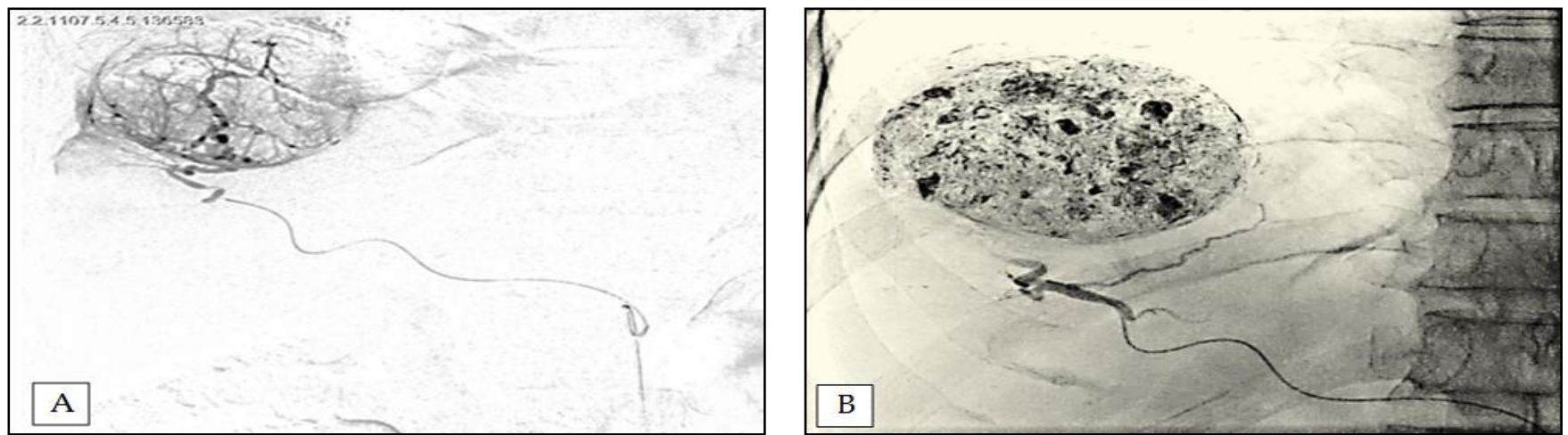

Figure-2: a) Super selective angiogram (with microcatheter) of segment VIII HCC supplying branch of right hepatic artery showed tumor blush, b) post conventional TACE super selective angiogram of segment VIII HCC supplying branch of right hepatic artery with microcathetr showed satisfactory flow stasis in tumor feeding artery and good lipoidal retention in tumor bed. 
(merit medical) mixed with $10 \mathrm{ml}$ of non-ionic contrast-ultravist (bayer) againmaking the total volume of $15 \mathrm{ml}$. in a few cases, only embolization was done with pva particles (45-150 and 255-350 micronsmerit medical) mixed with $10 \mathrm{ml}$ of non-ionic contrast-ultravist (bayer) again making the total volume of $15 \mathrm{ml}$ for embolization only. The chemotherapeutic emulsion was infused under fluoro guidance. Patients were randomly divided into two groups, $3 \mathrm{ml}$ of lidocaine $2 \%$ $(60 \mathrm{mg}$ ) was infused in group a just before the infusion of chemotherapeutic emulsion or pva particles and normal saline was infused in (group B) patients who did not receive intra-arterial lidocaine. In both groups, the procedure was concluded by infusing aliquots of polyvinyl alcohol particle (PVA) size of 45-150 micron till satisfactory flow stasis in tumor supplying hepatic artery.

Good hydration was assured for patients before and after procedure by iv normal saline infusion till the ability to drink.

Pain score was recorded using visual analog scale (vas) considering 1 as minimal discomfort and 10 as the most severe pain (fig-3). Pain scores were recorded

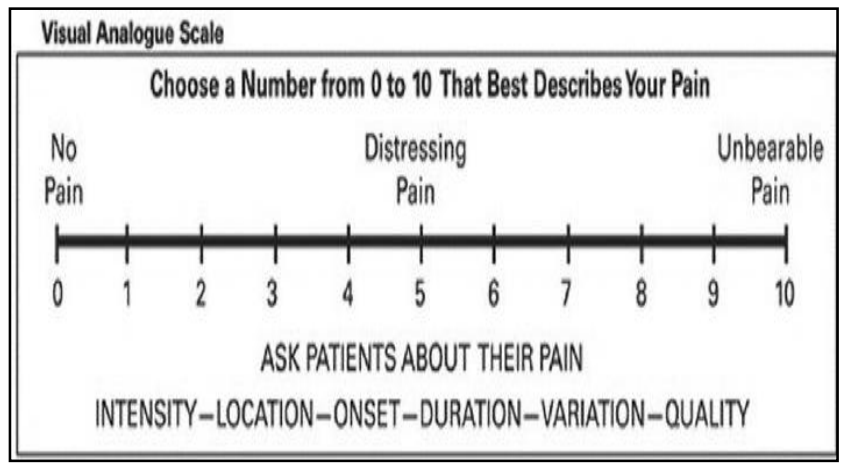

Figure-3: Visual analogue scale used to rate pain severity.

4 times on the procedure day and then two times per day. On the procedure, first recording was at the time of infusion of chemotherapy and embolizing material, second was immediately after the procedure, third reading was after 2 hours and last reading was in the evening about 6 hours after the procedure. Similar scores were taken twice a day for next two days, at 6am and $6 \mathrm{pm}$. Pain scores of $\geq 5$ was considered as significant pain that required analgesia. The scores were entered in the prescribed proforma. Wherever necessary, post procedure analgesia was provided by intravenous nalbuphine and both the mean dose and total dose of the drug were recorded. Hospital stay was also recorded for each patient in both groups. comparison of mean of the vas scores, requirement of nalbuphine and hospital stay between the two groups was done. Other symptomatic medication like ondansetron hydrochloride (onset) $4 \mathrm{mg}$ slowly intravenous infusion for nausea was given. Statistical analyses were performed with the independent sample t-test using SPSS. A $p$ value $<0.05$ was considered significant.

\section{RESULTS}

Sixty transarterial chemoembolization (TACE) procedures were carried out for 60 consecutive patients where $42(70 \%)$ patients were male and $18(30 \%)$ were female. Age of the patients ranged from 45 years to 85 years, mean age was $60.4 \pm 7.63$ years. Lidocaine group consisted of $30 \mathrm{TACE}$ procedures and every one of these patients received $60 \mathrm{mg}$ intra-arterial lidocaine during chemoembolization. Placebo group consists of 30 TACE procedures in 30 patients in whom intra-arterial lidocaine was substituted with normal saline solution. The patient demographic criteria, child pugh score, tumor size and doses of chemotherapeutic emulsion and amount of pva particles used were comparable without statistically significant difference. TACE technique was selective \& super selective or segmental and lobar for both lidocaine and placebo groups. The majority of patients had right lobe lesions. there was no recorded inadvertent embolization of the gall bladder supplying artery. There were no vascular complications during TACE procedure such as dissection or spasm of hepatic artery. The infused dose of chemotherapeutic emulsion till tumor bed saturation ranged from 0.5-1.0 of the prepared chemotherapeutic emulsion for the lidocaine group and from 0.5-0.9 of the emulsion in the placebo group, the dose difference was not statistically significant and so did the volume of the embolization particles (PVA). There were no recorded changes in blood pressure or arrhythmias in patients who received lidocaine. Moderate inter procedure pain was noticed in the entire lidocaine group $(n=30)$ with vas (visual analog scale) score of 4-6 compared to $5(16.6 \%)$ in the placebo group. Severe pain was noticed in $25(83.3 \%)$ of placebo group. The average periprocedural pain score was less in the lidocaine group than in the placebo group. The average vas was 5.06 versus 7.2 for lidocaine and placebo groups respectively $(p=0.037)$ (table). Post procedure pain was significantly lower in lidocaine group versus placebo group. vas score for pain in the lidocaine group was 2.7 \pm 0.520 and that for the placebo group was $4.2 \pm 0.761$, the difference is significant $(p=0.025)$. Vas score for pain on second day in the lidocaine group was $1.73 \pm$ 0.583 and that for the placebo group was $3.10 \pm 0.844$, 
the difference is significant $(p=0.018)$. The frequency of analgesic demands was higher in patients who did not receive lidocaine and total dose of nalbuphine in the lidocaine group was $4.96 \pm 0.764 \mathrm{mg}$ versus $8.3 \pm 1.34$ $\mathrm{mg}$ for patients in the placebo group $(p=0.036)$ (table).

Table: Difference between lidocaine and placebo group
as regards to pain score, nalbuphine requirement and
length of hospital stay.
\begin{tabular}{l|c|c|c} 
Lidocaine Groupplacebo & $\begin{array}{c}\text { Mean } \pm \\
\text { SD }\end{array}$ & $\begin{array}{c}\text { Mean } \pm \\
\text { SD }\end{array}$ & $\begin{array}{c}p \text { - } \\
\text { value }\end{array}$ \\
Group & $\begin{array}{c}5.06 \pm \\
\text { Mean of peri procedure }\end{array}$ & $7.4 \pm$ & 0.037 \\
visual analogue scale scores & 0.639 & 0.813 & 0.037 \\
\hline Mean of post procedure & $2.7 \pm$ & $4.2 \pm$ & 0.025 \\
visual analogue scale scores & 0.520 & 0.761 & \\
\hline Mean of 2nd day visual & $1.733 \pm$ & $3.10 \pm$ & 0.018 \\
analogue scale scores & 0.583 & 0.844 & \\
\hline Total dose of nalbin & $4.96 \pm$ & $8.30 \pm$ & 0.036 \\
(Nalbuphine) in mg & 0.764 & 1.34 & \\
\hline Length of hospital stay in & $0.90 \pm$ & $1.41 \pm$ & 0.002 \\
days & 0.203 & 0.373 & \\
\hline
\end{tabular}

Nausea was encountered in 27 (90\%) of patients belonging to the lidocaine group and it was $100 \%$ in those belonging to the placebo group. Incidence of post procedure fever was not statistically different in lidocaine and placebo groups, 4mg of ondansetron hydrochloride (onset), was slowly given as intravenous infusion to patients in $27(90 \%)$ of TACE procedures in the lidocaine group and was given to the entire placebo group patients. The average length of post procedure hospital stay was $0.9 \pm 0.203$ day and $1.41 \pm 0.373$ days for lidocaine and placebo groups respectively. that difference was statistically significant $(p=0.002)$.

\section{DISCUSSION}

Post embolization syndrome (PES) is experienced after TACE procedures in $80-90 \%$ of patients. It has widely variable manifestations but often includes pain, fever, nausea and vomiting. PES can last from a few hours to a few days 1. Although it is self-limiting condition, it is a major complication of hepatic TACE causing longer hospital stay ${ }^{9}$. As the large doses of intravenous narcotic analgesics needed to control the pain leads to altered mental status and respiratory depression; therefore intensive monitoring is required. Narcotic analgesics also potentiate severe post embolization nausea and vomiting ${ }^{11}$. The exact explanation of pain component of pes in TACE is not known but many hypothesis are postulated such as ischemia and transient swelling of liver parenchyma that stretches the liver capsule or accidental embolization of the arterial supply of gall bladder. Severe pain during the procedure can be explained by irritant effect of the che- motherapeutic emulsion on the hepatic artery branches $^{11,12}$. Direct irritation of the arterial wall by the chemotherapeutic emulsion is one of the theories of pain component of pes. Daniel et al found that post TACE pain was lower after the first session and this could be explained by lower dose of chemotherapy in the successive TACE sessions than that of the first TACE session $^{13}$. In super selective TACE, the arterial system which is in contact with irritant chemotherapeutic emulsion is at minimum \& the dose is lower than lobar TACE technique and carries less risk for non-target embolization of the gall bladder. Inadvertently embolized gall bladder artery was considered as one of the theories of pain component of pes. Contrary to daniel et al, patel et al found that repetition of TACE is not a predictor of pain component of pes as their hypothesis was that: ischemic pain was the main mechanism and vascular irritation by chemotherapeutic emulsion was not the major cause of pain ${ }^{14}$. In a study by coldwell et al excellent analgesia during hepatic TACE was achieved with a celiac plexus block. However, this method seems to be risky and time-consuming ${ }^{15}$. Intra-arterial lidocaine administration during TACE has been known not only for reduction of severity of the pain that is associated with TACE but facilitates faster recovery as well11,12. Lidocaine has been shown to help control the painful response to the injection of iodinated contrast material in peripheral arteries ${ }^{16,17}$. Molgaard et al11 studied the use of intra-arterial lidocaine in hepatic arterial branches prior to and during TACE. This resulted in a significant decrease in the amount of morphine (narcotic analgesic) required during the procedure as well as the need for subsequent postprocedure morphine drip. It remains clear that the sequelae of pain during and after TACE such as shallow respirations and paralytic ileus can complicate patient management. Therefore intra-arterial lidocaine administration is recommended because it is much easier and less time-consuming method than celiac plexus block. The mechanism of the analgesic effect of intra-arterial lidocaine in hepatic TACE is unclear. Hartnell et al ${ }^{12}$ suggested that lidocaine has a direct local effect after diffusion into the arterial wall \& liver parenchyma and this effect will be prolonged in tumors where blood flow is occluded preventing washout of the agent. Lee $e t$ al found that transcatheter administration of lidocaine immediately before infusion of chemotherapy had significantly better effect on pain control than after chemotherapy emulsion ${ }^{13}$. Lee et al found that patients who had received lidocaine by intra-arterial route during TACE procedure needed smaller doses of narcotic 
analgesics than those who had not received lidocaine $^{18}$. Different protocols for intra-arterial lidocaine administration were applied. Sharma et al ${ }^{19}$ used lidocaine intermittently during the TACE procedure. Hartnell et al ${ }^{12}$ injected lidocaine at varying intervals before and during TACE up to 4 times. The dose of lidocaine used in their study (maximum 105mg injected over 10-20 $\mathrm{min}$ ) was safe and effective. However Lee et al 18 concluded that intra-arterial administration of lidocaine just before TACE was much useful than after TACE as regards to pain control and post procedure requirement for narcotic.

In our study we used lidocaine as bolus rather than infusion; we found that $60 \mathrm{mg}$ of lidocaine was effective to alleviate pain during the procedure and reduced pain score and analgesic dose after the procedure. The post procedure pain control was significant in terms of low pain scores and smaller doses of analgesic requirement.

Superselective embolization is better than lobar TACE as regards to control of non-target embolization especially inadvertent embolization of the gall bladder which in some theory is the main cause of pain component of PES ${ }^{13}$. Super selective TACE reduces the number and length of arteries exposed to irritant chemotherapeutic emulsion which is one of the theories of pain component of PES. Super selective TACE is associated with reduction in the chemotherapeutic dose infused which is another advantage of superselective over lobar TACE technique in addition to much better tumor necrosis ${ }^{20}$. Lidocaine is metabolized by the liver and its half-life is about 2 and 2.5 hours with normal liver functions. Lidocaine metabolites and unchanged drug are excreted by the kidneys. Because of the rapid rate at which lidocaine is metabolized, any condition that affects liver function may alter lidocaine kinetics. The half-life may be prolonged two fold or more in patients with liver dysfunction such as cirrhotic patient with HCC. Entrapment of lidocaine in the vascular bed of liver tumor that is partially saturated by chemotherapeutic emulsion and infused embolization particle may prolong the duration of action of lidocaine but this cannot explain the extended duration of action for following few days ${ }^{21}$. Lidocaine has a potential antiinflammatory effect ${ }^{22}$ however; there is still a lack of well-designed studies to support this hypothesis. Interestingly, kogut et al ${ }^{23}$ found that prophylactic intraarterial administration of steroids in TACE procedures did not affect analgesic agent use and had a minor effect on antiemetic requirements.
In our study, although pre-procedure intra-arterial lidocaine administration improved pain component of pes, the length of hospital stay was slightly different in patients who received lidocaine versus patients who received placebo. Our results agreed with those of other authors ${ }^{24}$. The limitation of our study was that the number of patients and procedures were slightly small.

\section{CONCLUSION}

Intra-arterial administration of lidocaine just before infusion of embolization particles in dosesas low as $60 \mathrm{mg}$ is sufficient for pain control during TACE procedure and helps in pain control after the procedure. Furthermore in order to reduce the incidence of post-procedural pain and dose of post-procedural analgesics, we recommend routine pre-TACE administ-ation of lidocaine because post-procedural pain might develop even in patients who did not feel any pain during the TACE.

\section{CONFLICT OF INTEREST}

This study has no conflict of interest to be declared by any author.

\section{REFERENCES}

1. Alnagar M. Hepatocellular carcinoma treatment; non-surgical approaches. Int J Curr Res Rev 2016; 8 (16): 1-4.

2. Singal AG, El-serag HB. Hepatocellular carcinoma from epidemiology to prevention: translating knowledge into practice. Clin Gastroenterol Hepatol 2015; 13(12): 2140-51.

3. Lanza E, Donadon M, Poretti D, Pedicini V, Tramarin M, Rocallim, et al. Trans arterial therapies for hepatocellular carcinoma. Liver Cancer 2016; 6(1): 27-33.

4. Miki I, Murata S, Uchiyama F, Yasui D, Ueda T, Sugihara F, et al. Evaluation of the relationship between hepatocellular carcinoma location and transarterial chemoembolization efficacy. World J Gastroenterol 2017; 23(35): 6437-47.

5. Bannangkoonk, Hongsakulk, Tubtaweet, Piratvisuth T. Safety margin of embolized area can reduce local recurrence of hepatocellular carcinoma after super selective transarterial chemoembolization. Clin Mol Hepatol 2019; 25(1): 74-85.

6. Galanakis N, Kehagias E, Mathaiou N, Samonakis D, Tsetis D. Transcatheter arterial chemoembolization combined with radio frequency or microwave ablation for hepatocellular carcinoma: A review. Hepatic Oncol 2018; 5(2): 1-5.

7. Boily G, Villeneuve JP, Lacoursiere L, Chaudhury P, Couture F, Lapointe R, et al. Transarterial embolization therapies for the treatment of hepatocellular carcinoma: cepo review and clinical recommendations. HPB (Oxford) 2015; 17(1): 52-65.

8. Elbaz T, Kassas Me, Esmat G. Management of hepatocellular carcinoma: an updated review. J Cancer Thera 2013; (4): 536-45.

9. Lencioni R, Baere TD, Soulen MC, Rilling WS, Geschwind JFH. Lipiodol transarterial chemoembolization for hepatocellular carcinoma: a systematic review of efficacy and safety data. Hepatol 2016; 1(64) 106-16.

10. Dhand S, Gupta R. Hepatic transcatheter arterial chemoembolization complicated by post embolization syndrome. Semin Interv Radiol 2011; 28(2): 207-11. 
11. Lamy MN, Tan KT, Simons ME, Sniderman KW, Mironov O, Rajan DK. Intra-arterial lidocaine for pain control in uterine artery embolization: a prospective, randomized study. J Vasc Interv Radiol 2017; 28(1): 16-22.

12. Abusedera MA, Arafa UA, Ali EM. Transcatheter administration of buffered lidocaine for pain relief due to transarterial chemoembolization for HCC. Egypt J Radiol Nucl Med 2014; 45(2): 403408.

13. Daniel AL, James EG, Cheryl S, Barbara JR, Michael CS. Determinants of post embolization syndrome after hepatic chemoembolization. J Vasc Interv Radiol 2001; 12(3): 321-26.

14. Patel NH, Hahn D, Rapp S, Bergan K, Coldwell DM. Hepatic artery embolization: factors predisposing to post embolization pain and nausea. J Vasc Interv Radiol 2000; 11(4): c453-60.

15. Jung SN, Seon JI, Kim KS. The factors of pain and pain management after transarterial chemoembolization in patients with hepatocellular carcinoma. Asian Oncol Nurs 2017; 17(2): 107-15.

16. Guthaner DF, Silverman JF, Hayden WG, Wexler L. Intra-arterial analgesia in peripheral arteriography. Ajr Am Roentgenol 1977; 128(5): 737-39.

17. Biondi-Zoccai Gl, Moretti C, Omede P, Sciuto F, Agostoni P. Intra-arterial lidocaine versus saline to reduce peri-procedural discomfort in patients undergoing percutaneous trans-radial or trans-ulnar coronary procedures. Acta Cardiol 2011; 66(1): 9-14.
18. Kim MG, Kim HI, Yoon SH, Ko JG, Jeong JS, Han JS, et al. Evaluation of the effect of pain relief within the hepatic artery injection of lidocaine for reducing abdominal pains caused by TACE. J Korean Soc Interv Radiol 2011; 14(3): 199-205.

19. Sharma K, Gould J, Harbour J, Linette G. Hepatic arterial chemoembolization for management of metastatic melanoma. Am J Roentgenol 2008; 190(1): 99-104.

20. Golfieri R, Cappelli A, Cucchetti A, Piscaglia F, Carpenzano M, Peri E, et al. efficacy of selective transarterial chemoembolization in inducing tumor necrosis in small $(<5 \mathrm{~cm})$ hepatocellular carcinomas. Hepatol 2011; 53(5): 1580-89.

21. Eipe N, Gupta S, Penning J. Intravenous lidocaine for acute pain: an evidence-based clinical update. Br J Anaes 2016; 16(9): 292-98.

22. Caracas HC, Maciel JV, Martins PM, De Souza MM. The use of lidocaine as an anti-inflammatory substance: a systematic review. J Dent 2009; 37(2): 93-97.

23. Kogut MJ, Chewning RH, Harris WP, Hippe DS, Padia SA. Post embolization syndrome after hepatic transarterial chemoembolization: effect of prophylactic steroids on post procedure medication requirements. J Vasc Interv Radiol 2013; 24(3): 326-31.

24. Romano M, Giojelli A, Tamburrini O, Salvatore M. Chemoembolization for hepatocellular carcinoma: effect of intra-arterial lidocaine in peri- and post-procedural pain and hospitalization. Radiol Med 2003; 105(4): 350-55. 